\title{
Serotonin syndrome associated with methadone and milk thistle seeds: a case report
}

\author{
Andreja Celofiga ${ }^{1}$ \\ https://orcid.org/0000-0003-3979-9545 \\ Tomo Brus Hladen 1 \\ https://orcid.org/0000-0002-2215-3433 \\ ${ }_{1}^{1}$ Department of Psychiatry, University Medical Centre Maribor, Slovenia, Europe.
}

Received: 07/17/2019 - Accepted: 02/03/2020

DOl: 10.1590/0101-60830000000238

\begin{abstract}
Background: Serotonin syndrome is rarely, potentially life threatening condition, associated with use of serotonin acting medications and psychoactive drugs. In the majority of cases the symptoms occur soon after the initiation of a new drug or a change in the dose. Objective: To present a case report and to describe the possible mechanism of development of serotonin syndrome during the interactions between milk thistle seeds and methadone on hepatic cytochrome enzyme system P450. Methods: A case report of a young man on regular therapy with methadone, who develop a serotonin syndrome after ingestion a high dose of milk thistle seeds. Results: Commercial preparations of milk thistle include the extract silibinin, which exhibits no beneficial or harmful drug interactions at normal doses, but at higher concentrations it can lead to dose-dependent effects on methadone metabolism, through inhibition of CYP3A4 and P-glycoprotein. As a result, it may lead to enhanced serotonin re-uptake inhibition and increased serotonin activity. Discussion: Milk thistle is widely used and recommended for detoxification, but it may have serious and life threatening interactions with psychotropic drugs and psychoactive substances when used in high doses.
\end{abstract}

Celofiga A, Hladen TB / Arch Clin Psychiatry. 2020;47(3):85-6

Keywords: Serotonin syndrome, milk thistle, silymarin, methadone, interaction, cytochrome P450.

\section{Introduction}

Serotonin syndrome (SS) is a rare, potentially life threatening, condition, associated with the use of serotonin-acting medications and psychoactive drugs. The mechanism of the formation of SS is via postsynaptic hyperstimulation of 5-hydroxytryptamine (5-HT) $2 \mathrm{~A}$ and $1 \mathrm{~A}$ serotonin receptors in the central and peripheral nervous system. In the majority of cases the symptoms occur soon after the initiation of a new drug or a change in the dose. The classic triad of symptoms are: altered mental status (confusion, excitement, agitation, hallucinations), autonomic dysfunction (tachycardia, tachypnea, fever, diaphoresis) and neuromuscular abnormalities (tremor, hyperreflexia, clonus, myoclonus). SS is usually self-limited once the iniciting drug has been discontinued ${ }^{1,2}$.

\section{Case report}

A 51-years old male with psychoactive substance use disorder, who was on methadone substitution therapy ( $200 \mathrm{mg}$ daily) for 15 years, was admitted to a psychiatric unit for confusion and fluctuating levels of agitation. Before the admission he had been observed at the emergency department after experiencing restlessness, palpitations, dyspnea and resting tremor in the last 24 hours. ECG, laboratory tests and physical examination did not show any abnormalities, except high blood pressure $(200 / 120 \mathrm{mmHg})$, and tachycardia and tachypnea. He admitted using milk thistle seeds regularly for some time, but in the last three days he took a full fist of seeds daily in an attempt to "detoxify" his liver because of regular alcohol consumption. At the emergency department he was disoriented, confused and agitated, with hallucinations and he was consequently transferred to the psychiatric ward. Upon admission he was still confused, with high blood pressure and tachycardia. Restlessness, hallucinations and neuromuscular symptoms (tremor of the hands and jaw, myoclonus, hyperreflexia) were also observed. Alcohol and drug screening-tests were negative. Supportive measures and a sedative agents were introduced (lorazepam $2.5 \mathrm{mg}$ ). After three hours, the symptoms resolved completely.

\section{Discussion}

A possible mechanism of the development of SS was through interaction on the hepatic drug-metabolizing enzyme system cytochrome P450. Methadone is a serotonin re-uptake inhibitor, metabolized by cytochrome P450, mostly by CYP3A4 $4^{3}$. In vitro studies of methadone show a greater tendency toward serotonin reuptake inhibition compared with other opiates, which may explain methadone-mediated precipitation of SS ${ }^{4}$. Milk thistle is widely used for several medical conditions, especially for liver diseases. Commercial preparations of milk thistle include the extract silymarin, consisting of various flavonolignans, including silibinin, which have been shown to inhibit the in vitro activity of several cytochrome P450 enzymes including CYPP3A45. Silibinin exhibits no harmful drug interactions at normal doses (200-900 mg/day), but at higher concentrations it can lead to clinical important drug-drug interactions through inhibition of CYP3A4 and P-glycoprotein 6 . Results from in vitro studies on rats have indicated dose-dependent effects of silibinin on methadone metabolism, resulting in 50 to 100 percent reduction in methadone metabolism, which can lead to enhanced serotonin re-uptake inhibition and increased serotonin activity 7,8 .

\section{Conclusion}

The interactions between milk thistle seeds and methadone need further investigations and more attention is recommended in patients on long-therm methadone therapy using milk thistle to treat liver diseases.

\section{Conflict of interest}

Nothing to declare. 


\section{References}

1. Boyer EW, Shannon M. The Serotonin Syndrome. N Engl J Med. 2005;352(11):1112-20.

2. Gillman PK. A Review of Serotonin Toxicity Data: Implications for the Mechanisms of Antidepressant Drug Action. Biol Psychiatry. 2006;59(11):1046-51.

3. Ferrari A, Coccia CPR, Bertolini A, Sternieri E. Methadone - metabolism, pharmacokinetics and interactions. Pharmacol Res. 2004;50(6):551-9.

4. Gillman PK. Monoamine oxidase inhibitors, opioid analgesics and serotonin toxicity. Br J Anaesth. 2005;95(4):434-41.

5. Beckmann-Knopp S, Rietbrock S, Weyhenmeyer R, Böcker RH, Beckurts KT, Lang W, et al. Inhibitory Effects of Silibinin on Cytochrome
P-450Enzymes in Human Liver Microsomes. Pharmacol Toxicol. 2000;86(6):250-6.

6. Bhattacharya S. Phytotherapeutic properties of milk thistle seeds: An overview. J Adv Pharm Educ Res. 2011;1:69-79.

7. Pan PP, Wang J, Luo J, Wang SH, Zhou Y-F, Chen SZ, et al. Silibinin affects the pharmacokinetics of methadone in rats. Drug Test Anal. 2018;10(3):557-61.

8. Venkataramanan R, Ramachandran V, Komoroski BJ, Zhang S, Schiff PL, Strom SC. Milk Thistle, a Herbal Supplement, Decreases the Activity of CYP3A4 and Uridine Diphosphoglucuronosyl Transferase in Human Hepatocyte Cultures. Drug Metab Dispos. 2000;28(11):1270-3. 